\title{
THREE-DIMENSIONAL PIEZOELECTRIC ACCELEROMETER FOR MEASURING DYNAMIC PARAMETERS OF MOVING OBJECTS
}

\author{
Tofig M. Mansurov, Azerbaijan Technical University, Baku, Azerbaijan \\ Chingiz P. Aliyev, Ministry of Defense Industry, Baku, Azerbaijan
}

DOI: $10.36724 / 2664-066 X-2020-6-6-10-16$

\begin{abstract}
Analysis of structural construction of existing three-dimensional accelerometers is carried out, in which due to the opposite direction of sensitivity vectors and direction of measured inertial force, they allow to measure only linear inertia and accelerations varying in relatively small limits. On the other hand, due to the presence of elements in them that create electromagnetic fields, their construction is somewhat complicated. In addition, in known accel-erometers, due to the absence of a measurement object position sensor and an electronic control circuit, the func-tionality of the accelerometer is limited, Due to lack of integrator for acceleration integration, speed measurement is not provided, Due to rigid attachment of piezoelectric sensing elements through their bases, during measurement they generate interference signals, The amplitude of which exceeds the amplitude of the useful signal, which does not ensure reliability of the measured acceleration or speed and due to the presence of sensitive piezoelectric ele-ments, Having higher rigidity and requiring relatively large mechanical forces for generation of signals during mo-tion of acceleration and speed measurement object in automatic mode, which reduces sensitivity of accelerometer.

Invention proposes new design and control scheme of three-dimensional piezoelectric accelerometer for measure-ment of dynamic parameters of moving objects in automatic mode. Wherein providing the position sensor of the
\end{abstract}

moving measurement object with a three-axis signal detection unit to determine a direction of motion with meas-urement of acceleration or linear velocity when the measurement object moves along the coordinate axis; With the help of differential operational amplifiers through pulse generators and integrators, Speed measurement is provided by damping piezoelectric elements when the measurement object moves along one of the coordinate axes; Across the other two axes, the generated interference signals by amplitude are significantly reduced by their redemption; Having a piezoelectric element in the structure in the form of twolayer flat plates with an excitation section and a sensor section; Which generates signals at fast-changing acceleration and speed of moving object, amplitude of signal and sensitivity increases in 4-5 due to generation of signal by sensitive elements in vibration excitation mode created in accelerometer.

Mathematical basis for accelerometer control circuits, mathematical model of its functioning is developed, applica-tion of which increases sensitivity of measurement of linear accelerations and speeds of moving objects and expands functional capabilities.

KEYWORDS: accelerometer, piezoelectric element, sensitivity vector, inertial force, linear acceleration, velocity, vibration measurement

Information about authors

Tofig M. Mansurov, Professor of department "Telecommunications and Information Security", Doctor of Technical Sciences, Azerbaijan Technical University, Baku, Azerbaijan

Chingiz P. Aliyev, Director General of Production Association "UFUG", Ministry of Defense Industry, Baku, Azerbaijan 


\section{Introduction}

The relevance of oscillatory processes occurring study in moving objects is associated with the development of a three-dimensional accelerometer for measuring dynamic parameters of moving objects in real time. The use of information-measuring systems makes it possible to automate process of measuring the dynamic parameters of moving objects, which facilitates accounting of rare events that strongly affect the measurement results $[2,3,6,7]$.

For this purpose, three-coordinate position sensors are installed in the measuring devices, which perceive movements of measurement object along three coordinates of a rectangular coordinate system, placed on moving objects and converting a mechanical quantity into a corresponding electrical signal. This signal goes to the recording device. Piezoelectric vibration measuring transducers, which are generator-type sensors, which convert vibration acceleration into a proportional electrical signal, are mainly used as such position sensors.

The accelerometer consists of a sensitive mass fixed in an elastic suspension, under the influence of the apparent acceleration, a deviation from the initial position occurs, proportional to this acceleration [1-3]. Accelerometers are devices with one degree of freedom and contain an inertial mass, flat springs as an elastic support system, and a damping device. Position sensors detect the movement of the inertial mass relative to the measurement object and convert it into a proportional electrical signal. In this regard, another component of the accelerometer is a displacement detector capable of measuring the amplitudes of vibration vibrations in a certain range or linear acceleration. The output signal of the accelerometer, which is proportional to the value of the acceleration of mechanical vibrations, is converted into an electrical signal by means of a meter.

The object of research is a three-dimensional piezoelectric accelerometer for measuring the dynamic parameters of moving objects in an automatic mode.

The subject of research is study of basic principles and patterns of increasing sensitivity and reliability and expanding the functionality of a three-dimensional piezoelectric accelerometer for measuring dynamic parameters of moving objects in an automatic mode.

The aim of the work is to improve the accuracy and reliability of measurements, sensitivity and expand the functionality of a three-dimensional piezoelectric accelerometer for measuring dynamic parameters of moving objects with a rapidly changing direction of movement of measurement object along the three axes "XYZ" of a rectangular coordinate system in automatic mode.

\section{Formulation of the problem}

To measure the dynamic parameters of moving objects in automatic mode, piezoelectric accelerometers are used as a working element of the accelerometer. The use of piezoelectric accelerometers is associated with their advantage over other known types of accelerometers. Piezoelectric accelerometers have a wide operating frequency range, active conversion, have a linear amplitude characteristic, high resistance to external influences. The absence of moving parts in them ensures high reliability and durability; they can be implemented in a small-sized design with high manufacturability in production.

In known accelerometers, the sensitivity vector and direction of measured inertial force have opposite directions, which makes it possible to measure only linear inertia and acceleration within small limits. Such accelerometers do not allow measuring the speeds of moving objects in real time. Due to the presence of elements that create electromagnetic fields, their design becomes more complicated and requires additional measures to exclude the influence of these electromagnetic fields [7-10].

In this regard, it becomes necessary to develop a threedimensional piezoelectric accelerometer for measuring the dynamic parameters of moving objects, a mathematical basis for the construction and a mathematical model of its functioning, in which the polarization vector of the piezoelectric element is directed along the axis of sensitivity of the accelerometer, i.e. direction of the vector of linear acceleration of moving objects. Along with this, below is the classification of accelerometers, their characteristics and parameters measured by the accelerometer, objective function is formulated.

\section{Development of target function}

To assess the effectiveness of a three-dimensional piezoelectric accelerometer for measuring the dynamic parameters of moving objects, determined by an increase in accuracy and reliability of measurements, sensitivity and functionality expansion of this accelerometer with a rapidly changing direction of object measurement movement along the three axes "XYZ" of a rectangular coordinate system, an objective function is formulated in an automatic mode. Accuracy of the accelerometer depends on sensitivity of sensors and dynamic range, and sensitivity, i.e. amplitude with which the device emits an electrical signal, which is considered main parameter when choosing a measuring device. The accuracy of data obtained during the measurement depends on it. The dynamic range of vibrations captured by accelerometer is determined by vibration energy, which is in a very narrow frequency range. Therefore, it is necessary to take into account that the vibration measurement process is performed with very small or very large amplitudes of acceleration and, accordingly, speed.

Improving the efficiency of this accelerometer operation consists in solving two main tasks, i.e. in the development of objective function and determination of optimal values of its arguments, which would ensure the operation of accelerometer with a rapidly changing direction of movement of measurement object along the three axes "XYZ" of a rectangular coordinate system in automatic mode.

Guided by the above principles, the target function of a three-dimensional piezoelectric accelerometer can be determined in the following form:

$$
E_{\text {TPA }}=\left\{\min \left[t_{i z}, \delta_{i z}, P_{p o m}\right], \max \left[\tau_{l_{1}}, A_{c h}, D_{i z}\right]\right\},
$$


where $t_{i z}-$ measurement time; $\delta_{i z}-$ measurement error; $P_{\text {pom }}$ - noise level along two other coordinates, along which measurements are not being performed at a given time; $\tau_{1_{1}}$ - accelerometer speed; $A_{c h}-$ the sensitivity of the accelerometer, i.e. amplitude, starting from which the accelerometer emits an electrical signal and is considered the main parameter when choosing a measuring device; $D_{i z}$ - dynamic range of measurement of an accelerometer, which is determined by the upper and lower values of vibrations that the accelerometer is capable of detecting and is in a very narrow frequency range.

\section{Development of a three-dimensional piezoelectric accelerometer for measuring the dynamic parameters of moving objects}

To expand the functionality, increase the accuracy, sensitivity and reliability of measuring dynamic parameters of moving objects relative to the coordinate axes "XYZ" of a rectangular coordinate system in the automatic measurement mode with a rapidly changing direction of movement of the measurement object, a design scheme for the accelerometer for measuring the dynamic parameters of moving objects in automatic mode has been developed "and its control scheme, which is shown in Fig. $1[1,4,5]$.

The developed accelerometer contains a piezoelectric element along each coordinate, made in the form of a two-layer flat plate, glued or welded together by wide edges having an excitation section $-1,2,3$ and a sensitive element section - 4,5,6, isolated from each other by insulating grooves $-7,8,9$, and the piezoelectric elements are damped relative to the longitudinal coordinate axes through "P" - shaped flat springs - 10,11,12, while the measurement object -13 is equipped with a threecoordinate position sensor -14 , the electrodes -15 of which are connected to the first input of the first differential operational amplifier - 16, to the second input of which the output of the sampling unit is connected -17 axes of the rectangular coordinate system "XYZ", the first -18 , the second -19 , the third -20 , the outputs of the first differential operational amplifier along three coordinate axes are connected to the inputs of the threshold device - 21, which, depending on the set operation threshold does not pass the generated interference signals in two other coordinates to the output, the recognition unit -22 coordinate signals with the ability to process a random sequence, the outputs of the threshold device are connected to the inputs of the memory unit -23 , which is connected to the control code generator -24 and to the control unit -25 , the outputs of which along the three axes "XYZ" are connected to the control inputs of the triggers - 26,27,28, выходы которых соединены к входам электронных ключей 29,30,31, signal inputs and outputs of which are respectively connected to the inputs of the alternating voltage source - 32,33,34 coordinate axes "XYZ" and to the excitation electrodes of piezoelectric elements, and the electrodes of the sensitive elements of the coordinate axes "XYZ" are connected to the inputs of differential operational amplifiers - 35.36, 37 coordinate axes
"XYZ", the first outputs of which through pulse shapers $38,39,40$ are connected to the first digital indicators $41,42,43$, and the second outputs of differential operational amplifiers through pulse shapers - 44,45,46 and integrators $-47,48,49$ are connected to the inputs of the second digital indicators - 50.51.52. Inertial masses $53,54,55$ are mechanically connected with piezoelectric sensing elements, piezoelectric elements are installed on "P" -shaped flat springs. Each of the piezoelectric sensing elements, located along the XYZ axes, together with the mechanically connected inertial mass, is placed inside a separate protective casing $-56,57,58$.

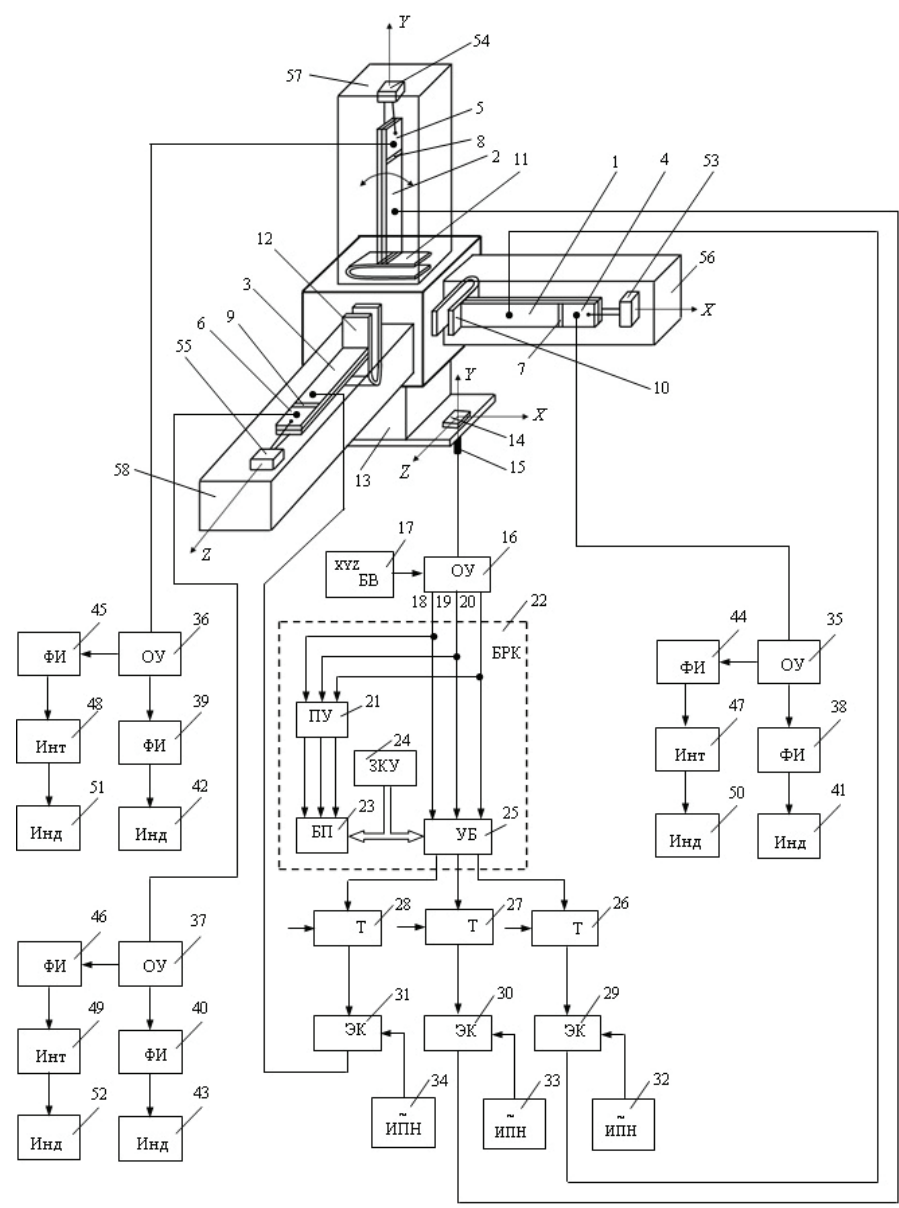

Figure 1. Diagram of the design of a three-dimensional piezoelectric accelerometer for measuring the dynamic parameters of moving measurement objects with a control circuit

\section{Operating principle}

The principle of the accelerometer operation is based on the fact that, depending on required direction of measurement object movement - 13 at the beginning in the sampling block -17 , the initial state is set along one of the XYZ axes of a rectangular coordinate system [5, 6].

When the measurement object moves $-13 \mathrm{~V}$ along the "X" axis, first the three-coordinate position sensor - 14 perceives the movement of the measurement object - 13 and signals from its output are fed to the input of the first differential operational amplifier - 16. Amplified signals, passing through the threshold device - 21, which depending on the set triggering threshold, it does not pass the generated interference signals along two other 
coordinates to the output, they enter the coordinate recognition unit -22 , which are fed into the memory unit - 23. The signals from the output of control code generator -24 are fed to the input of memory unit -23 and the controlled unit -25 , from output of which the signals are recognized by their amplitude, by their frequency or by their average value in allocated frequency band. Since the maximum signal amplitude occurs along the "Y" axis, since the direction of sensitivity axis in three-coordinate sensor - 14 along the "Y" axis of rectangular coordinate system coincides with direction of measurement object movement - 13 along the " $\mathrm{X}$ " axis, signals from the output of coordinate recognition unit 22 axis " $Y$ " is fed to the trigger control input -27 , from the output of which signals sent to the control input of electronic key - 30. In this case, the signals from output of AC voltage source - 33 are fed to the signal input of electronic switch -30 , from the signal output of which voltage is fed to the electrodes of excitation section -2 piezoelectric elements, which create alternating bending vibrations in it, the direction of which coincides with the direction of sensitivity axis vector of section sensing element -5 .

With the linear movement of the measurement object - 3 , depending on the change in speed in the direction along the " $\mathrm{X}$ " axis, the inertial mass - 54, due to its inertia, lags behind or ahead of the body of the measurement object -13 , which creates the effect of inertial forces on the sensitive element -5 , leading it to bending deformations that generate signals proportional to the varying acceleration. These signals are fed to a differential operational amplifier - 36 .

Strengthening, these signals through the pulse shaper 39 are fed to the digital indicator -42 , showing the measured acceleration of the linear motion of the measurement object 13 , and the signals passing through the pulse shaper -45 , the integrator - 48 and the digital indicator - 51 shows the measured speed of the measurement object -13 .

The measurement of the dynamic parameters of the movement of the measurement object -13 relative to the other two axes "Y" and "Z" of the rectangular coordinate system is carried out in an automatic mode of operation similarly in the above sequence. With the help of a threecoordinate position sensor -14 , a differential operational amplifier - 16 and a coordinate recognition unit -22 , automatic switching of the coordinate axes "XYZ" is carried out depending on the change in the direction of movement of the measured object - 13, inertial masses $53,54,55$ are mechanically connected with piezoelectric sensitive elements. Each of the piezoelectric sensing elements, located along the XYZ axes, together with an inertial mass mechanically connected to it, is located inside a separate protective casing - 56,57,58

\section{Mathematical basis of the accelerometer functioning}

In the developed accelerometer, it is proposed to use a piezoelectric element as a working element, made in the form of two-layer flat plates along each coordinate axis $\mathrm{X}$, $\mathrm{Y}$ and $\mathrm{Z}$, glued or welded together by wide edges having an excitation section and a sensitive element section, depending on the change in direction of movement measurement object.

The use of a piezoelectric element as a working (converting) organ is associated with its advantages listed above.

When the measurement object moves along one of the coordinate axis, an effect is created on sensitivity sections of accelerometer piezoelectric element, measured in units of inertial gravity, and as a result, the voltage components obtained from electrodes of piezoelectric element are created.

The moment of piezoelectric element inertia is associated with geometric shape of inertial mass and is determined as follows [4-6]:

$$
i=l_{2} \cdot l_{3}^{3} / 12,\left(\mathrm{~m}^{4}\right)
$$

where $l_{2}-$ piezoelectric element width $(\mathrm{m}) ; l_{3}-$ piezoelectric element thickness (m).

The stiffness of accelerometer piezoelectric element is determined by following formula:

$$
C=l_{1} / 3 \cdot E_{ю} \cdot i,(\mathrm{~N} / \mathrm{m})
$$

where $l_{1}$ - length of piezoelectric element $(\mathrm{m}) ; E_{ю}-$ Young's modulus (modulus of elasticity) $\left(\mathrm{N} / \mathrm{m}^{2}\right) ; i-$ moment of inertia of the piezoelectric element $\left(\mathrm{m}^{4}\right)$.

According to expression (2) in known accelerometer, the piezoelectric element has a relatively high stiffness value [1] and therefore, to remove a signal with a voltage of $0.5 \mathrm{mV}$ from the electrodes of a sensitive piezoelectric element, the value of the linear inertial force should be 5-6 times higher than the value of this force, acting on a sensitive piezoelectric element. Since the value of the linear inertial force is at a low sensitive area, it allows you to measure relatively smaller values of this force.

By differentiating the velocity vector of free end of piezoelectric element by time vector, one can determine the acceleration of free end of piezoelectric element by following formula:

$$
\vec{i}=\frac{d \vec{v}}{d t}=\frac{d^{2} r}{d t}
$$

where $\vec{v}$ - piezoelectric element free end speed $(\mathrm{m} / \mathrm{s}) ; t-$ time (s); $r$ - trajectory of movement.

If we take into account the trajectories of measurement object along one of the coordinate axis, then at some point in time $-t_{0}$ trajectory vector $\vec{r}(t)=\vec{r}_{0}$ and time dependence of acceleration vector of free end of piezoelectric element $\vec{i}=\varphi(t)$.

Then integrating formulas (3) on the time interval from $t_{0}$ to $t$ we obtain the following dependences to determine $\vec{v}-$ the speed of free end of piezoelectric element and $\vec{r}(t)-$ motion path vector:

$$
\vec{v}(t)=\vec{v}_{0}+\int_{t_{0}}^{t}(t) d t,
$$




$$
\vec{r}(t)=\vec{r}_{0}+\left(t-t_{0}\right) \vec{v}_{0}+\int_{t_{0}}^{t} \int_{t_{0}}^{t} \vec{i} d t^{2}
$$

If the time vector $\vec{i}$ does not change, then the given motion of the measurement object is considered uniformly accelerated and, transforming formulas (4) and (5), we obtain expressions for determining the speed of the free end of the piezoelectric element and the vector of the motion trajectory:

$$
\begin{aligned}
& \vec{v}(t)=\vec{v}_{0}+\left(t-t_{0}\right) \cdot \vec{i}, \\
& \vec{r}(t)=\vec{r}+\left(t-t_{0}\right) \cdot \vec{v}_{0}+\frac{\left(t-t_{0}\right)^{2}}{2} \cdot \vec{i} .
\end{aligned}
$$

Depending on the purpose of accelerometer, mathematical basis makes it possible to determine necessary nodes when building a control circuit for a three-coordinate piezoelectric accelerometer for measuring dynamic parameters of moving objects in an automatic mode.

\section{Mathematical model of functioning}

Mathematical model is built according to the following sequence. First, to calculate the main parameters of piezoelectric element, we determinedisplacement amplitude of free end of piezoelectric element, made in form of two-layer flat plates with a length $l_{1}$ with longitudinal vibrations of first mode along its length according to following formula:

$$
\Delta_{P A}=\frac{2 T_{c} \cdot l_{1}}{\pi \cdot E_{u}}=\frac{2 E_{\sim} \cdot d_{31} \cdot Q_{m} \cdot E_{u} \cdot l_{1}}{\pi}, \mathrm{m}
$$

where $T_{c}-$ maximum allowable mechanical stress $\left(\mathrm{N} / \mathrm{m}^{2}\right)$.

The maximum allowable mechanical stress arising in a deformable piezoelectric element during the movement of the measurement object is determined by following expression:

$$
T_{c}=E_{\sim} d_{31} \cdot Q_{m} \cdot E_{u}, \mathrm{~N} / \mathrm{m}^{2}
$$

where $E_{\sim}-$ voltage of electric field applied to the plates of piezoelectric element made in form of two-layer flat plates $(\mathrm{V} / \mathrm{m}) ; d_{31}-$ piezoelectric module for piezoceramics TsTBS-3 (piezoelectric ceramics from lead zirconate-titanate) $(\mathrm{m} / \mathrm{V}) ; Q_{m}-$ mechanical quality factor, determined experimentally from amplitudefrequency (resonance) characteristics of piezoelectric element (Fig. 2); $E_{u}-$ Young's modulus for a piezoelectric element of the TsTBS-3 brand $\left(\mathrm{N} / \mathrm{m}^{2}\right)$.

If we do not take into account the structural losses, then we can obtain dependence for maximum permissible linear velocity of free end of piezoelectric element:
$V_{p r . d o p}=\frac{4 F_{c} \cdot V_{z v}}{\pi \cdot l_{1} \cdot l_{2} \cos \alpha \cdot E_{u}}=\frac{4 F_{c}}{\pi \cos \alpha \cdot Z_{0}}=\frac{4 F_{c} V_{z v}}{\pi \cos \alpha \cdot E_{u}},(\mathrm{~m} / \mathrm{s})$

where $Z_{0}=l_{1} \cdot l_{2} \cdot \sqrt{\rho \cdot E_{u}}$ - wave resistance; density of CBTS-3 material $\left(\mathrm{kg} / \mathrm{m}^{3}\right)$.

The resonant frequency of a piezoelectric element made in the form of two-layer flat plates can be determined by following formula:

$$
f_{p}=V_{z v} / 2 \cdot l,(\mathrm{kHz})
$$

where $V_{36}$ - longitudinal wave propagation speed $(\mathrm{m} / \mathrm{s})$.

The speed of the working end of a loaded piezoelectric element made in form of two-layer flat plates is determined as follows:

$$
V_{p k n}=\frac{U_{\sim v o z b} \cdot l_{2} \cdot d_{31} \cdot E_{u}}{R_{m p} \cdot \cos \alpha},(\mathrm{m} / \mathrm{s})
$$

where $\alpha-$ the angle of inclination of piezoelectric element made in form of two-layer flat plates to working body (rad); resistance to mechanical losses.

Mechanical loss resistance is defined as follows:

$$
R_{M P}=\omega \cdot M / Q_{m},(\mathrm{~N} \mathrm{~s} / \mathrm{m})
$$

The average linear speed of working body movement is determined by following formula:

$$
V_{p o}=\Delta_{P A} \cos \alpha \cdot k \cdot(\mathrm{m} / \mathrm{s})
$$

Taking into account the complex linear nature of friction value, correction factor for average linear speed of movement of working body is determined by formula:

$$
k=V_{p o} / V_{p k n}
$$

where $V_{p o}$ - linear speed of working body movement $(\mathrm{m} / \mathrm{s}) ; V_{р к н}-$ speed of the end of working body of the loaded piezoelectric element made in form of two-layer flat plates $(\mathrm{m} / \mathrm{s})$.

Taking into account the losses associated with the action of a constant clamping force of the piezoelectric element on working element, speed of the end of working element of loaded piezoelectric element is determined as follows:

$$
V_{p k}=V_{p k n} / 2,(\mathrm{~m} / \mathrm{s})
$$

The nominal moment acting on piezoelectric element is determined by following formula:

$$
M_{H}=F_{T} \cdot R,(\mathrm{Nm})
$$


where $F_{T}-$ maximum tangential force on the piezoelectric element $(\mathrm{N}) ; R$ - bending radius of the piezoelectric motor $(\mathrm{m})$.

The maximum tangential force on a piezoelectric element is determined by following expression:

$$
F_{T}=U_{v o z b} \cdot l_{2} \cdot d_{31} \cdot E_{u} \cos \alpha .(\mathrm{H})
$$

where $U_{v o z b}-$ AC source voltage $(\mathrm{V}) ; l_{2}$ - piezoelectric element thickness $(\mathrm{m}) ; d_{31}-$ piezoelectric module of piezoceramics $(\mathrm{m} / \mathrm{V}) ; E_{u}-$ Young's modulus (modulus of longitudinal elasticity), i.e. physical quantity characterizing the ability of a material to resist stretching, compression in bending $\left(\mathrm{N} / \mathrm{m}^{2}\right)$.

Taking into account equation (18) in equation (17), we obtain the following expression for a piezoelectric element made in form of two-layer flat plates glued or welded together by wide edges having an excitation section and a sensitive element section:

- $\quad$ - for a piezoelectric element made in the form of two-layer flat plates:

$$
M_{1}=U_{v o z b} \cdot l_{2} \cdot d_{31} \cdot E_{u} \cos \alpha \cdot R,(\mathrm{Nm})
$$

- - for "P" -shaped flat springs, with which the piezoelectric elements are damped relative to the longitudinal coordinate axes:

$$
M_{2}=F_{p r} \cdot R,(\mathrm{Nm})
$$

where $F_{p r}-$ clamping force of "P"-shaped flat springs $(\mathrm{N})$.

The moment created by the tangential force and the force of "P" -shaped flat springs acting on a piezoelectric element made in form of two-layer flat plates is determined as follows:

$$
M=M_{1}+M_{2},(\mathrm{Nm})
$$

The time required for starting and establishing mechanical vibrations of a piezoelectric element made in form of two-layer flat plates is determined as follows:

$$
t_{\text {nyck }}=1,47 \cdot \frac{Q_{m}}{f_{p}}, \text { (c). }
$$

where $f_{p}-$ resonant frequency of the supply voltage $(\mathrm{Hz}) ; Q_{\mu}-$ mechanical figure of merit of piezoelectric element.

The mechanical figure of piezoelectric element merit made in form of two-layer flat plates is determined from the amplitude-frequency characteristic (fig. 2) according to following formula:

$$
Q_{m}=f_{p} / \Delta f
$$

where $\Delta f-$ bandwidth; $f_{p}-$ mechanical resonance frequency $(\mathrm{Hz})$.

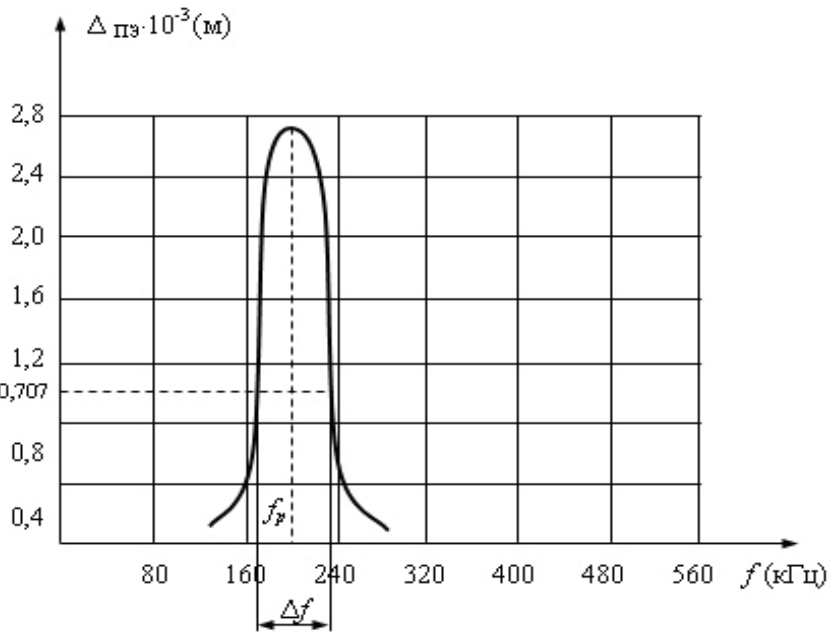

Figure 2. Amplitude-frequency (resonance) characteristic of piezoelectric element

The speed of accelerometer depends on frequency of mechanical resonance of piezoelectric element made in form of two-layer flat plates, parameters of electrical circuit of power supply and is determined by following formula:

$$
\tau_{l_{1}}=1 / 2 \cdot f_{p} \cdot(\mathrm{Hz})
$$

As follows from the above analytical dependences, which were used to determine the main parameters of accelerometer, coincide with experimental data and discrepancy between them is $1-3 \%$.

\section{Conclusion}

Thus, the developed three-coordinate piezoelectric accelerometer for measuring the dynamic parameters of measurement moving objects in automatic mode has the following advantages over known accelerometers:

1. 1. In the proposed accelerometer for measuring the dynamic parameters of a moving measurement object in automatic mode, the supply of a position sensor of measurement object using a signal recognition unit of three coordinate axes "XYZ" of a rectangular coordinate system ensures determination of movement direction, i.e. coordinate axis $\mathrm{X}, \mathrm{Y}$ or $\mathrm{Z}$ with measurement of acceleration or linear velocity when measurement moving object along this coordinate axis.

2. 2. In the proposed accelerometer, connection of second outputs of differential operational amplifiers through pulse shapers and integrators connected to inputs of second digital indicators provides measurement of movement speed for measurement object relative to three axes "XYZ" of rectangular coordinate system.

3. 3. In the proposed accelerometer, due to the damping of piezoelectric elements with the help of "P" shaped flat springs along three axes "XYZ" of trectangular coordinate system when e measurement object moves along one of the coordinate axes, along other two axes, generated noise signals in amplitude are significantly reduced by their suppression, since the directions of mechanical vibrations that create amplitudes 
of interference are located along the axes of rectangular coordinate system of piezoelectric elements.

4. 4. In the proposed accelerometer, the presence in construction of piezoelectric element of a section for excitation of mechanical vibrations, as well as a section of a sensitive element that generates signals with rapidly changing acceleration and speed of a moving object of measurement, the signal amplitude and sensitivity increase 45 times due to the generation of a signal by sensitive elements in vibration excitation mode created in the accelerometer.

Comparative analysis shows that the above advantages of developed accelerometer directly affect the achievement of specified technical result, i.e. allow to expand functionality, increase the sensitivity and reliability of process of measuring dynamic parameters of a moving object in measurement relative to the "XYZ" axes of a rectangular coordinate system in automatic mode with a rapidly changing direction of measurement object movement.

\section{References}

1. A.A. Brickichev. Piezoelectric accelerometer. Patent of the Russian Federation No. 2400760. http://www.findpatent.ru/patent/240/2400760.html C FindPatent .ru - patent search, $2012-2015$.
2. V.V. Jancic. Piezoelectric vibration measuring converters (accelerometers). Rostov-on-Don: News of the Southern Federal University, 2010. 304 p.

3. V.M. Sharapov, M.P. Musienko, E.V. Sharapova Piezoelectric sensors. Ed. V.M. Sharapova. Moscow: Technosphere, 2006. 632 p.

4. T.M. Mansurov, Ch.P. Aliyev. Three-coordinate accelerometer for measurement of acceleration and speed of measurement object in automatic mode. Bulletin of the Eurasian Patent Office (EAPV) of the Eurasian Patent Organization (priority of application № 201700327/31). 2018. No. 9. P. 125. 28.09.2018

5. T.M. Mansurov, Ch.P. Aliyev. Accelerometer for measurement of accelerations and speeds of moving objects in automatic mode. Journal of Khmelnitskii National University. Kharkov, CNU. No. 5, 2018. P. 78-83.

6. Yu.I. Iorish. Vibrometriya. Second edition, revised and supplemented. Moscow: State Scientific and Technical Publishing House of Machine-Building Literature, 1963. 771 p.

7. M.D. Genkin, A.G. Sokolova. Vibrations in Engineering. Moscow: Mechanical engineering, 1981. Vol. 5. Measurements and tests. Ed. M.D. Genkin. 1981. 496 p.

8. K. Brindley. Transmitters: Reference: Per. Moscow: Energoatomizdat, 1991. 144 p.

9. G. Vigleb. Sensors. Device and application. Moscow: World, 1989. $196 \mathrm{p}$. 